\title{
Performance of Long Modules of Silicon Microstrip Detectors
}

G. Baricchello ${ }^{a}$, A. Cervera-Villanueva ${ }^{b}$, D.C. Daniels ${ }^{c}$, E. do Couto e Silva ${ }^{b}$, L. Dumps ${ }^{b}$, M. Ellis ${ }^{d}$, D. Ferrère ${ }^{b, 1}$, J.J. Gomez-Cadenas ${ }^{b}$, M. Gouanère $^{e}$, J.A. Hernando ${ }^{f, 2}$, W. Huta ${ }^{b}$, J.M. Jimenez ${ }^{b}$, J. Kokkonen ${ }^{b}$, V.E. Kuznetsov ${ }^{g}$, L. Linssen ${ }^{b}$, B. Lisowski ${ }^{h, 3}$, Ö. Runolfsson ${ }^{b}$, F.J.P. Soler ${ }^{d}$, D. Steele ${ }^{i}$, M. Stipcević ${ }^{j}$, M. Veltri ${ }^{k}$.

\begin{abstract}
This note describes the performance of modules assembled with up to twelve silicon microstrip detectors. These modules were built for the instrumented Silicon Target (STAR) that has been installed in the NOMAD spectrometer. Laboratory and test beam results are compared with model predictions. For a module of nine detectors, test beam results indicate a signal-to-noise ratio of 19 , a hit finding efficiency of $99.8 \%$ and a spatial resolution of $6.0 \mu \mathrm{m}$. Laboratory measurements indicate that modules of twelve detectors exhibit a signal-to-noise ratio of the order of 16 .
\end{abstract}

a. University of Padua, Padua, Italy.

b. CERN, Geneva, Switzerland.

c. Harvard University, Cambridge, MA, USA.

d. University of Sydney, Sydney, Australia.

e. LAPP, Annecy, France.

$f$. University of Valencia, Valencia, Spain.

g. Joint Institute for Nuclear Research, Dubna, Russia.

$h$. Dortmund University, Dortmund, Germany.

$i$. University of Lausanne, Lausanne, Switzerland.

j. Rudjer Bošković Institute, Zagreb, Croatia.

$k$. University of Urbino, Urbino and INFN, Florence, Italy.

Submitted to Nuclear Instrumentation and Methods.

\footnotetext{
${ }^{1}$ Now at University of Geneva, Geneva, Switzerland.

${ }^{2}$ Now at University of California at Santa Cruz, California, USA.

${ }^{3} \mathrm{On}$ leave of absence from Institute of Electron Technology, Warsaw, Poland.
} 


\section{Introduction}

One of the most interesting current problems in particle physics is the possibility that neutrinos have non-vanishing masses and that there are oscillations among the different families. At present, two experiments, CHORUS and NOMAD $[1,2]$ are searching for the exclusive $\nu_{\mu}\left(\nu_{e}\right) \leftrightarrow \nu_{\tau}$ oscillation modes in the CERN-SPS beam. To understand the design of a large surface silicon tracker for a future $\nu_{\mu}\left(\nu_{e}\right) \leftrightarrow \nu_{\tau}$ oscillation experiment $[3,4,5,6,7]$ we have built an instrumented silicon target (NOMAD-STAR) which has been taking data in the NOMAD spectrometer since May of 1997.

Tau identification in NOMAD is based exclusively on the use of kinematical techniques. The NOMAD-STAR detector will allow a precise determination of the event vertex, and therefore of the impact parameter of tau decay candidates. The NOMAD apparatus has been described elsewhere [2] and a description of the NOMAD-STAR detector can be found in reference [8].

NOMAD-STAR consists of modules of $72 \mathrm{~cm}$ length read out from only one end by low-noise electronics. To evaluate the performance of these very long modules, a test beam experiment and extensive laboratory studies were conducted. The main results are presented in this paper.

The organization is as follows: in section 2 we describe the NOMAD-STAR modules (ladders). Section 3 discusses the sources of noise in a silicon detector and presents a comparison between analytical calculations and laboratory measurements. Section 4 describes the test beam set-up. Test beam results and studies on charge sharing between strips are discussed in section 5. Conclusions are presented in section 6 .

\section{NOMAD-STAR modules}

Figure 1 illustrates the assembly of a module (ladder). Twelve silicon detectors are glued to a thin kapton foil which electrically isolates them from a conducting carbon-fiber backbone of about $0.5 \mathrm{~cm}$ thick. One of the ends of the ladder is glued to an aluminum support that contains a hybrid printed circuit board and fixing and alignment holes. The readout chips are mounted on the hybrid board.

\subsection{Silicon detectors}

The silicon microstrip detectors ${ }^{4}$ are similar to those used in the DELPHI experiment [9]. These are single-sided, $33.5 \mathrm{~mm} \times 59.9 \mathrm{~mm}$, with strip and readout pitches of $25 \mu \mathrm{m}$ and $50 \mu \mathrm{m}$, respectively. The $p^{+}$strips are implanted in a high resistivity $300 \mu \mathrm{m}$-thick $n$-type substrate, and are AC coupled to the electron-

\footnotetext{
${ }^{4}$ Manufactured by Hamamatsu Photonics, Japan.
} 


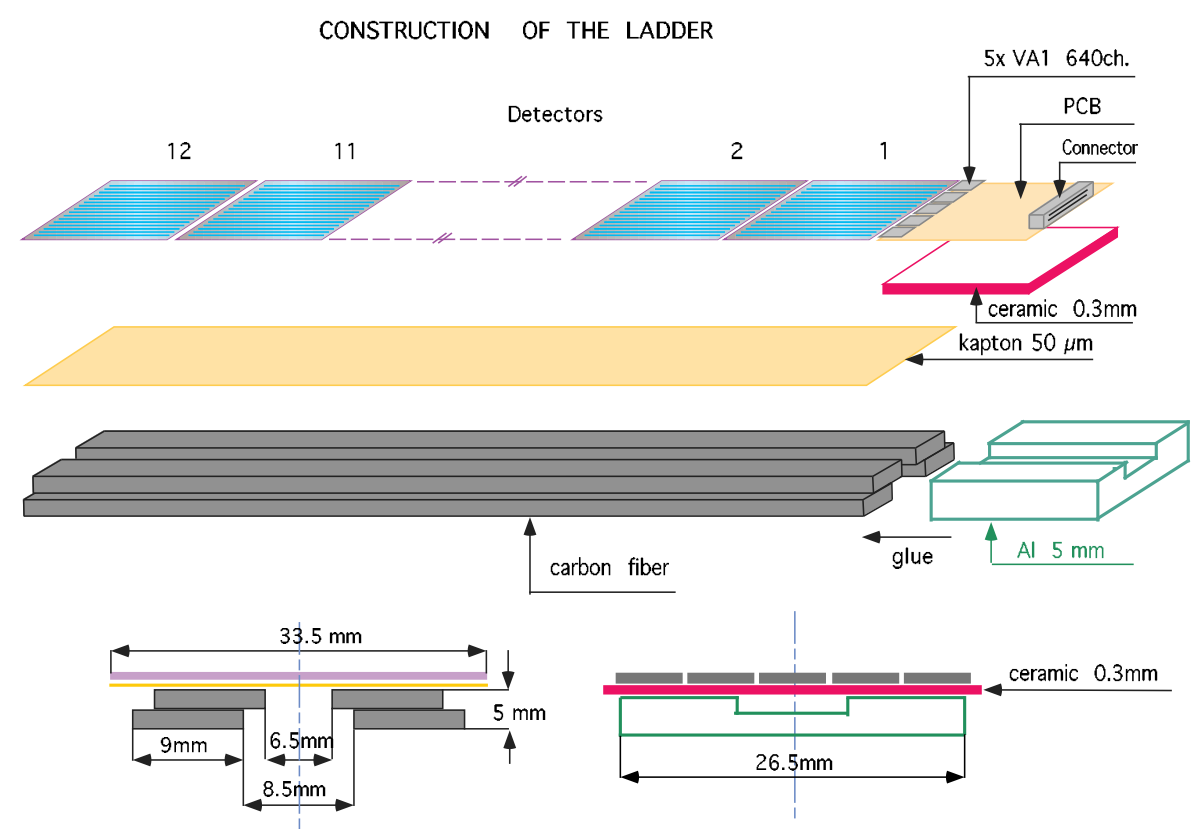

Figure 1: Schematical drawing of a ladder.

ics via a silicon oxide layer. The biasing of the strips is done via the FOXFET mechanism [10]

\section{$2.2 \quad$ Readout electronics}

We have used the VA1 readout chip $^{5}$. It consists of 128 charge sensitive, lowpower $(1.2 \mathrm{~mW} /$ channel $)$ and low-noise preamplifiers followed by $\mathrm{CR}-\mathrm{RC}$ shapers, track-and-hold circuitry, output multiplexing and a multiplexing calibration circuit.

Each ladder is read out by five VA1 chips mounted on a printed circuit board ${ }^{6}$. The sequential output signals are amplified on a repeater card which also drives the clock signals to the hybrid and allows adjustment of various voltages controlling the operation of the VA1 readout chip.

After a trigger, the sequential readout is performed by activating the output shift registers, using a clock operating at a frequency of $1 \mathrm{MHz}$, and shift-in/shiftout signals.

\footnotetext{
${ }^{5}$ It is a commercial version of the VIKING chip [11] distributed by IDE AS, Norway.

${ }^{6}$ Also distributed by IDE AS, Norway.
} 


\section{$3 \quad$ Noise studies}

The spatial resolution and the hit finding efficiency depend on the strip pitch and on the signal-to-noise ratio. To estimate the expected signal-to-noise ratio we use a simple mathematical model that takes into account the dominant sources of noise in the amplifier.

\subsection{Sources of noise}

We consider the following sources of noise:

- shot-noise created by leakage current in the detector,

- thermal noise from the biasing resistors of the detector,

- series-resistance noise in the detector and in the front of the input-transistor of the pre-amplifier,

- flicker noise in the input transistor,

- channel thermal noise in the input transistor,

- bulk series-resistance noise in the input transistor.

All the above noise sources are white except the flicker noise which has a 1/f dependence on the frequency. The first two sources introduce parallel noise, and the remaining ones are considered to be in series with the input transistor.

For low leakage currents, the parallel noise is mainly a Johnson-type thermal noise generated in the biasing resistors of the detectors and/or the feedback resistor of the preamplifiers. The series noise can be classified as external or internal to the preamplifier. The only external source to the preamplifier is a Johnsontype noise due to the resistance of the metal strip connected to the input of the preamplifier. The internal sources originate from fluctuations in the drain current (Johnson-type).

\subsection{Equivalent noise charge}

Noise figures are usually expressed in terms of Equivalent Noise Charge (ENC). This parameter relates the root mean squared $(\mathrm{rms})$ noise voltage at the output of the shaper directly to the signal strength at the input. A detailed discussion is provided in the Appendix.

Table 1 displays the mathematical formulae used to calculate the contributions from the different sources of noise.

The noise contribution due to the readout chip, $\mathrm{ENC}_{V A 1}$, is:

$$
\mathrm{ENC}_{V A 1}=\mathrm{ENC}_{1 / f} \oplus \mathrm{ENC}_{c h} \oplus \mathrm{ENC}_{b u l k} .
$$




\begin{tabular}{|l|l|c|}
\hline Source of noise & Type & $\mathrm{ENC}\left[\mathrm{rms} e^{-}\right]$ \\
\hline Leakage current & parallel & $\mathrm{ENC}_{\text {leak }}=\frac{e}{q} \sqrt{\frac{q I_{\text {leak }} T_{p}}{4}}$ \\
\hline Polarization resistors & parallel & $\mathrm{ENC}_{\text {res }}=\frac{e}{q} \sqrt{\frac{k T T_{p}}{2 R_{p}}}$ \\
\hline Metal strip & series & $\mathrm{ENC}_{m s}=\frac{e C_{t}}{q} \sqrt{\frac{k T R_{m s}}{6 T_{p}}}$ \\
\hline Transistor flicker noise & series & $\mathrm{ENC}_{1 / f}=\frac{e C_{t}}{q} \sqrt{\frac{F_{k}}{2 W L_{e f f}}}$ \\
\hline Transistor channel & series & $\mathrm{ENC}_{c h}=\frac{e C_{t}}{q} \sqrt{\frac{k T}{3 g_{m} T_{p}}}$ \\
\hline Transistor bulk-resistance & series & $\mathrm{ENC}_{b u l k}=\frac{e C_{t}}{q} \sqrt{\frac{R_{b u l k} \eta^{2} k T}{2 T_{p}}}$ \\
\hline
\end{tabular}

Table 1: Summary of the sources of noise (see definitions in the Appendix). 
where the mathematical formulae for $\mathrm{ENC}_{1 / f}, \mathrm{ENC}_{c h}$ and $\mathrm{ENC}_{b u l k}$ are shown in Table 1. Instead of calculating the last three sources of noise, we use the measured values for the $\mathrm{ENC}_{V A 1}$ as an input for our model.

Tests with passive elements indicate that optimal noise performance is at shaping times between 2 and $3 \mu \mathrm{s}$. As shown in Figs. 2a our measurements are in good agreement with the specifications from the manufacturer. The parametriza-
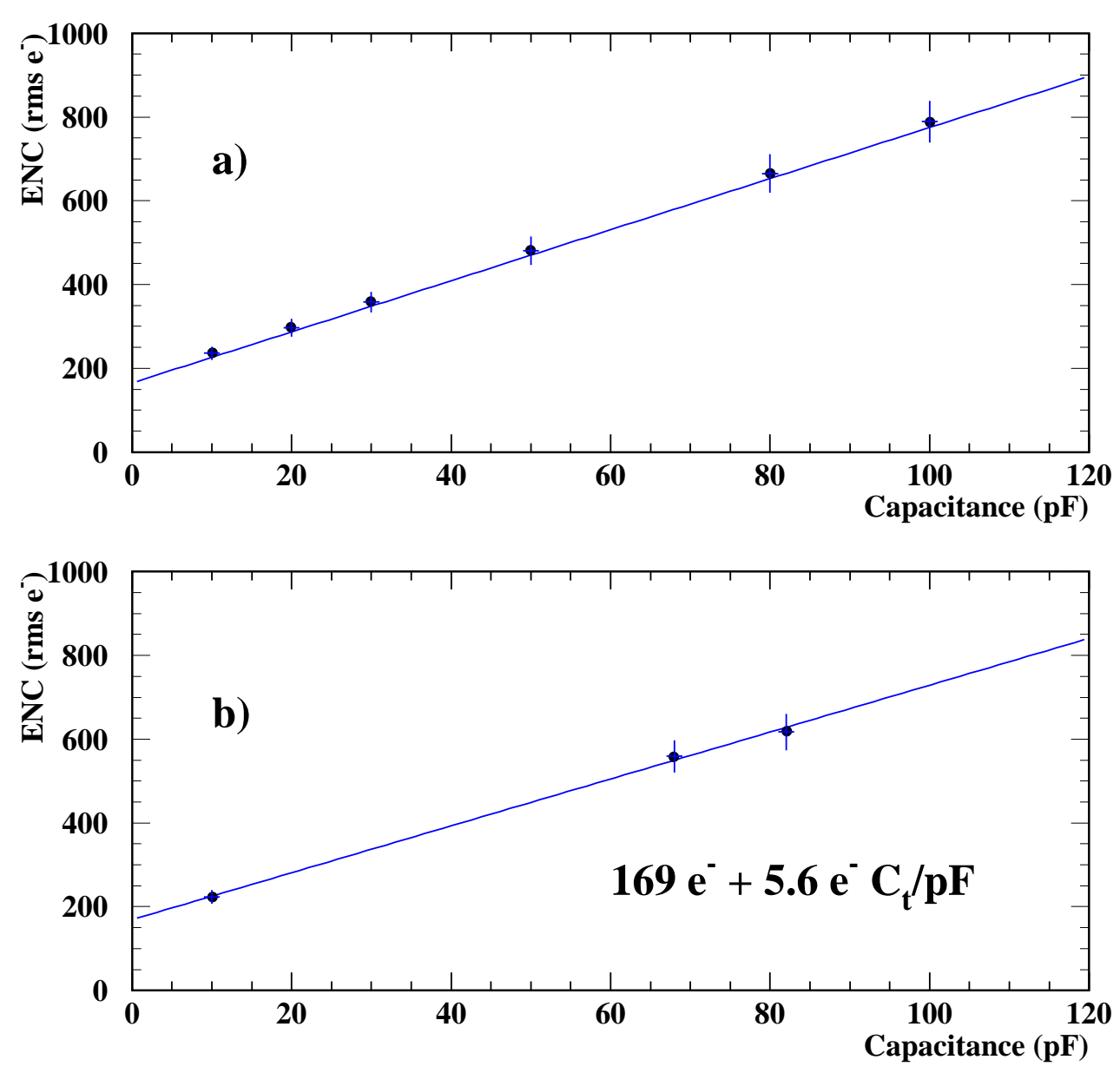

Figure 2: Noise performance of the VA1 readout chip for: a) $\tau_{p}=2 \mu$ s measured in the laboratory (data points) overlaid with the specifications from the manufacturer (solid line), b) $\tau_{p}=3 \mu$ s measured in the laboratory (data points) overlaid with best fit (solid line).

tion for $\mathrm{ENC}_{V A 1}$ is obtained from the fitted curve of Fig. 2b:

$$
\mathrm{ENC}_{V A 1}=169 e^{-}+5.6 \mathrm{e}^{-} \mathrm{C}_{t} / \mathrm{pF}
$$


where $\mathrm{C}_{t}$ is given in units of picofarads.

\subsection{Total noise for the NOMAD-STAR ladders}

The four relevant sources of noise are $\mathrm{ENC}_{V A 1}, \mathrm{ENC}_{\text {leak }}, \mathrm{ENC}_{r e s}$ and $\mathrm{ENC}_{m s}$.

To calculate the expected noise for the ladders, we used the parameters shown in Table 2.

\begin{tabular}{|c|c|c|}
\hline Parameter & $\begin{array}{c}\text { Measured Value } \\
\text { for a detector }\end{array}$ & $\begin{array}{c}\text { Calculated Value } \\
\text { for a ladder }\end{array}$ \\
\hline Interstrip capacitance & $1.2 \mathrm{pF} / \mathrm{cm}$ & $86.4 \mathrm{pF}$ \\
\hline Backplane capacitance & $0.15 \mathrm{pF} / \mathrm{cm}$ & $10.8 \mathrm{pF}$ \\
\hline Total capacitance $\left(C_{t}\right)$ & $1.35 \mathrm{pF} / \mathrm{cm}$ & $97.2 \mathrm{pF}$ \\
\hline Strip resistance $\left(R_{m s}\right)$ & $31.5 \Omega / \mathrm{cm}$ & $2268 \Omega$ \\
\hline Leakage current per strip $\left(I_{\text {leak }}\right)$ & $0.08 \mathrm{nA}$ & $0.96 \mathrm{nA}$ \\
\hline FOXFET dynamical resistance & $>500 \mathrm{M} \Omega$ & - \\
\hline Total parallel resistance $\left(R_{p}\right)$ & - & $29.4 \mathrm{M} \Omega$ \\
\hline
\end{tabular}

Table 2: Summary of the parameters used in the model.

The very low current of the detectors and the high dynamical resistance provided by the FOXFET are important to minimize the parallel noise. The total leakage current for most of the detectors used is below $100 \mathrm{nA}$, which corresponds to an average current of less than $100 \mathrm{pA}$ through individual FOXFET bias gates. An attempt to measure the dynamical resistance was done by injecting current into a test contact on a given diode strip and measuring the voltage drop using a semiconductor parameter analyzer ${ }^{7}$. The gate-drain voltage difference was kept at zero. The results are shown as closed circles in Figure 3. Taking into account the average leakage current per diode strip one obtains the curve represented by the open circles. Combining the results shown in Fig. 3 and the instrumental errors (controlled by calibrations), we estimate the effective average resistance for the FOXFET gate to be $>500 \mathrm{M} \Omega$. For the noise calculations due to the dynamical resistance of the FOXFET we used the value of $500 \mathrm{M} \Omega$.

The total parallel resistance $\left(R_{p}\right)$ is obtained by:

$$
\frac{1}{R_{p}}=\frac{N}{R_{p o l}}+\frac{1}{R_{f}}
$$

where $N$ is the number of detectors in a ladder, $R_{\text {pol }}$ is the dynamical resistance of the FOXFET for each detector and $R_{f}$ is the feedback resistor of the preamplifier ${ }^{8}$.

\footnotetext{
${ }^{7}$ HP4155 from Yokogawa-Hewlett-Packard, Ltd., Japan.

${ }^{8}$ It is expected to be at least $50 \mathrm{M} \Omega$ [12]; in our calculations we assumed $R_{f}=100 \mathrm{M} \Omega$.
} 


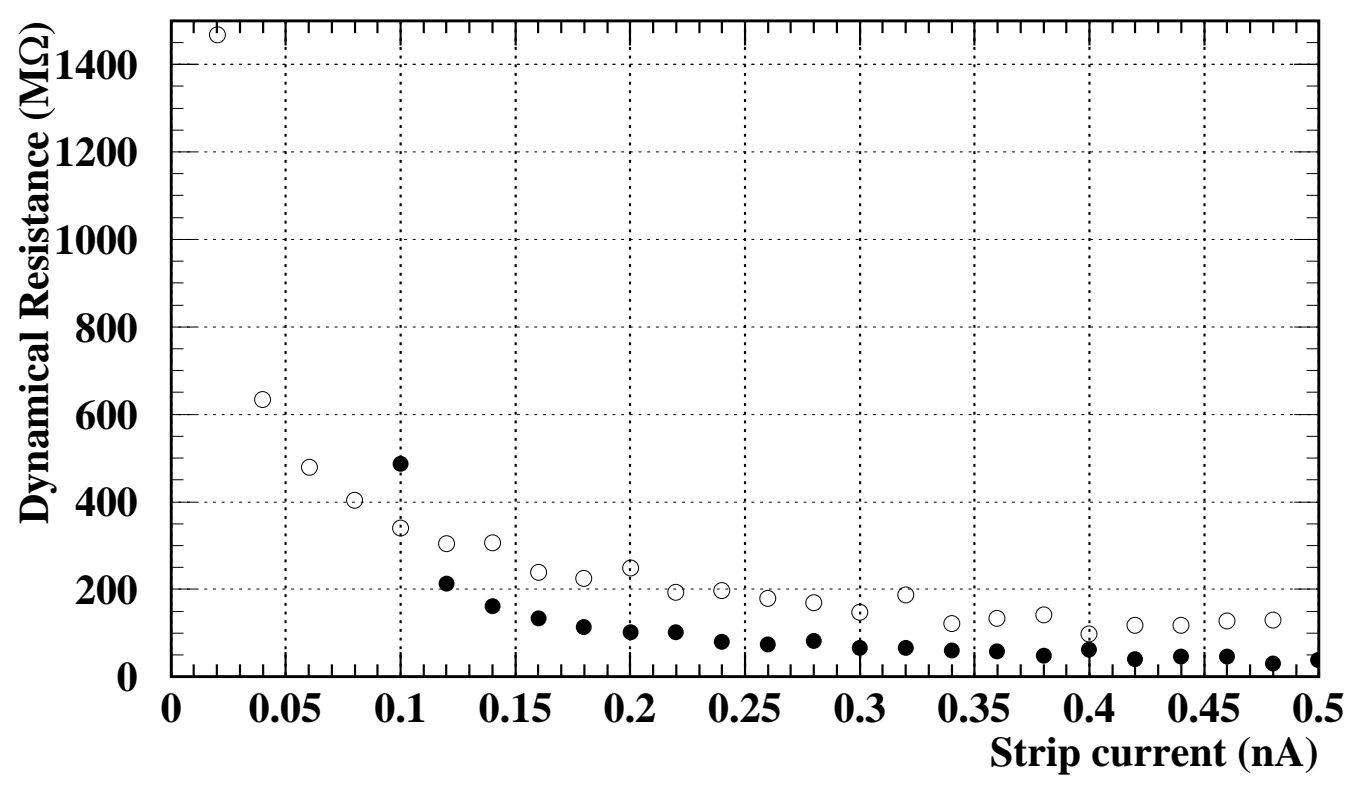

Figure 3: Dynamical resistance versus the strip current. For the closed (open) circles the strip current corresponds to the injected current (injected plus average leakage current).

The numbers from Table 2 and the equations displayed in Table 1 are used to estimate $\mathrm{ENC}_{\text {leak }}, \mathrm{ENC}_{\text {res }}, \mathrm{ENC}_{m s}$.

The series noise depends on the total detector capacitance (dominated by the interstrip capacitance) and on the total resistance. The readout chip was chosen to minimize the contribution of the capacitance, leaving only the relatively high trace resistance as the dominant source of noise. In Fig. 4 we show that the dominant noise contribution comes from the metal strip resistance $\left(\mathrm{ENC}_{m s}\right)$. This can be reduced if one decreases the resistance per unit length by increasing the width and/or the thickness of the aluminum strips.

\subsection{Response tests with a radioactive source}

We compare the predictions of the model with measurements performed using a radioactive ruthenium source ${ }^{9}$. To predict the noise performance of a typical ladder we add the sources of noise in quadrature

$$
\mathrm{ENC}_{\text {ladder }}=\mathrm{ENC}_{V A 1} \oplus \mathrm{ENC}_{\text {leak }} \oplus \mathrm{ENC}_{\text {res }} \oplus \mathrm{ENC}_{m s}
$$

Prototype ladders were built in several steps and the signal-to-noise ratio was measured when ladders consisted of 1, 2, 3, 4, 9, 10, 11 and 12 detectors (a detector has a length of $6 \mathrm{~cm}$ ). For the measured data points, the signal-to-noise ratio is defined as the fitted peak position when a Landau distribution convolved

\footnotetext{
${ }^{9}$ Electrons emmitted with maximum energy of about $3.5 \mathrm{MeV}$.
} 


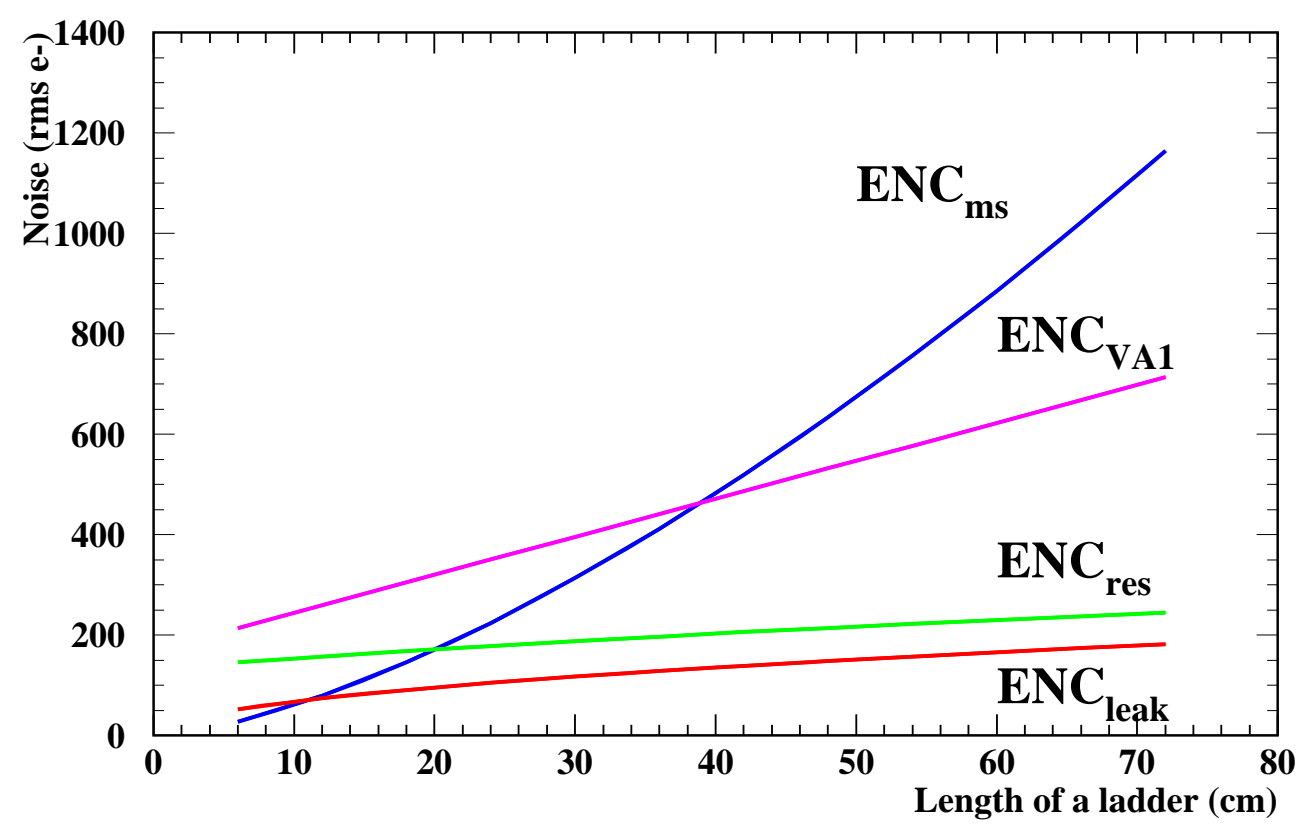

Figure 4: Sources of noise versus the length of a ladder from theoretical predictions.

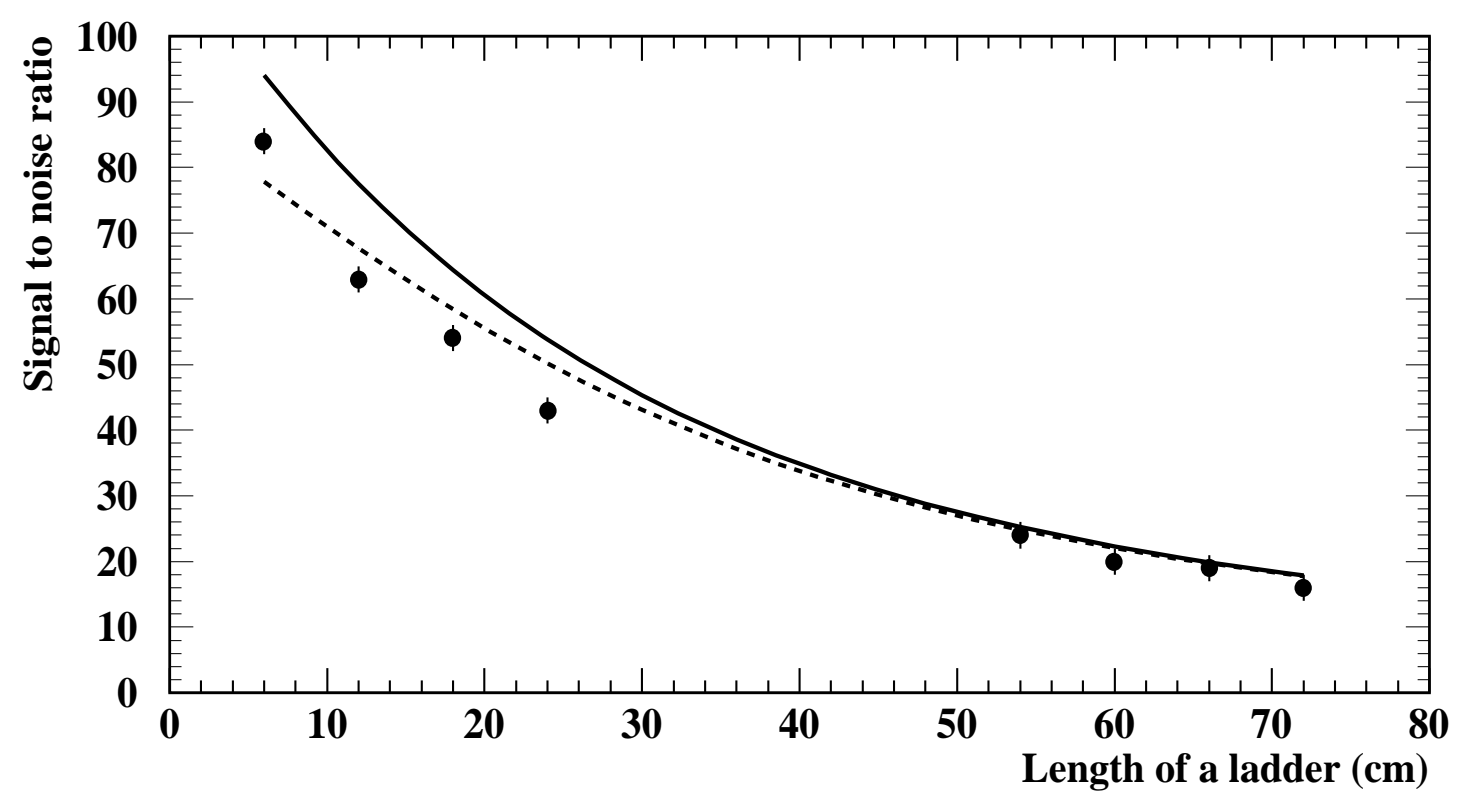

Figure 5: Calculated signal-to-noise ratio (solid curve) versus the length of the ladder. The laboratory measurements are represented by the closed circles. The dashed curve correponds to the calculated signal-to-noise ratio assuming an additional noise contribution from the hybrid (see text). 
with a Gaussian is used to fit the charge distribution for the cluster. For the model assumed, a minimum ionizing particle traversing a detector creates 25,000 electron-hole pairs, therefore the signal-to-noise ratio is

$$
\frac{S}{N}=\frac{25,000}{E N C_{\text {ladder }}}
$$

The solid curve in Fig. 5 shows the expected signal-to-noise ratio for a ladder as a function of its length. The laboratory measurements are represented by the closed circles. The disagreement between the calculated curve and the experimental data can be explained by an additional source of noise due to the hybrid. This additional source of noise is estimated to be of the order of 180 rms electrons. The dashed curve shows the expected behavior when this noise is added in quadrature to that of equation (2). We have built fifty ladders of twelve detectors and their measured signal-to-noise ratio is given in Fig. 6.

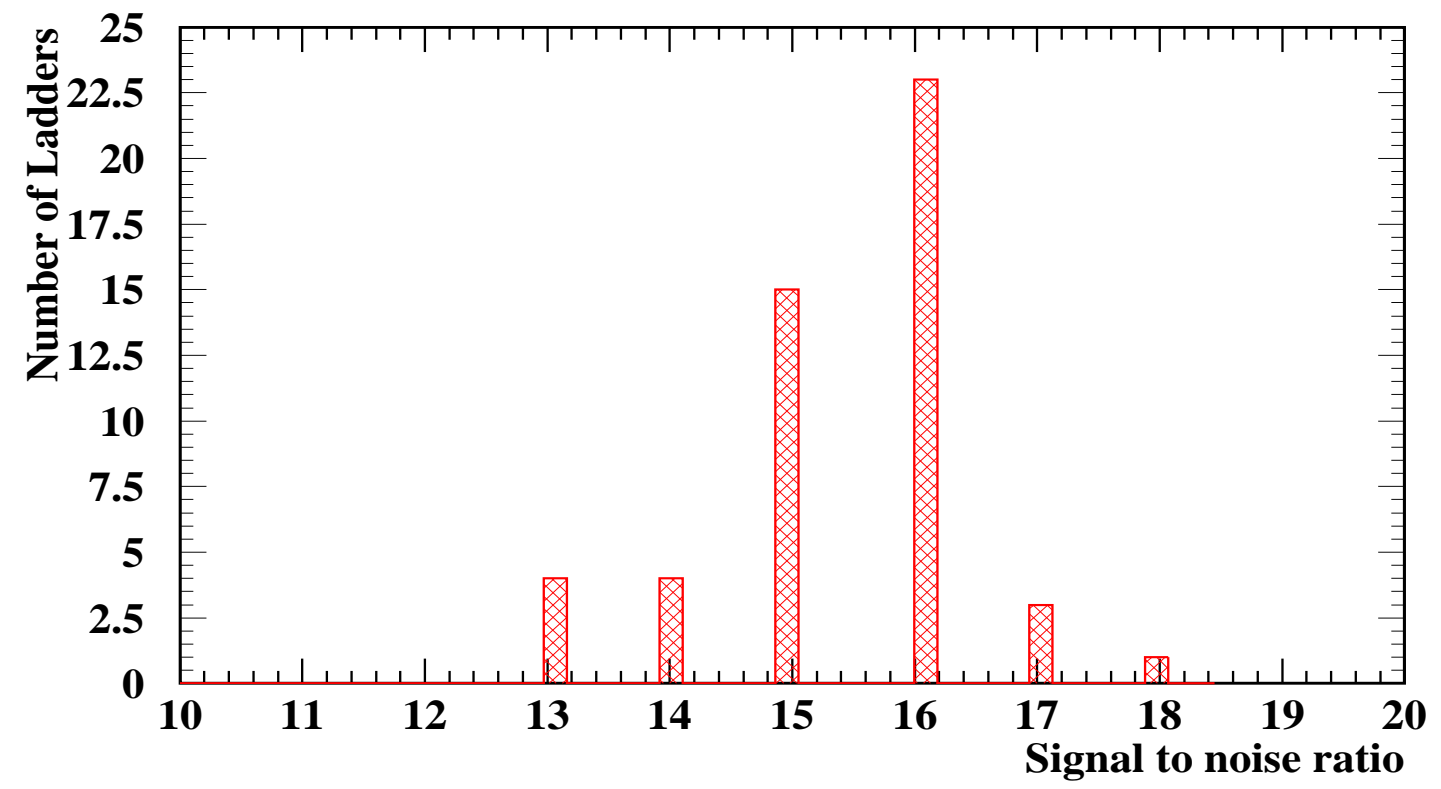

Figure 6: Signal-to-noise distribution from laboratory measurements for 50 ladders of 12 detectors.

\section{Test beam}

A ladder of 9 detectors was tested in a particle beam of the CERN SPS. The beam consisted of negative pions with momentum above $100 \mathrm{GeV} / \mathrm{c}$, thus the multiple scattering was considered negligible. 


\subsection{Experimental set-up}

The experimental set-up consisted of a silicon telescope mounted onto an optical bench fixed to a marble table and connected to a standalone VME data acquisition system. This set-up has been described at length elsewhere [13].

The silicon telescope is shown schematically in Fig. 7. It consisted of four reference planes of silicon detectors. The telescope planes consisted of two singlesided detectors oriented perpendicular to each other. The most upstream plane was defined as the first plane. The first detector in each plane was oriented with the strips along the $x$ direction while the second detector had its strips along the $y$ direction. Trigger signals were provided by scintillator counters connected in coincidence.

The ladder under test was mounted at one of the ends of the telescope ${ }^{10}$ (plane 5 of Fig. 7).

\section{Data analysis}

The data analysis aims to determine the spatial resolution of the detector and to study its hit-finding efficiency and the signal-to-noise ratio.

The spatial resolution is obtained by subtracting in quadrature the extrapolation errors of tracks reconstructed by the telescope from the residuals. A residual is defined as the difference between the impact point obtained from the charge collected on the detector strips and the point in which the extrapolated track reconstructed by the telescope intersects the plane of the detector.

As described in section 5.1 the extrapolation errors are obtained by finding the trajectory of the particle using the reference detectors. The distribution of residuals depends on the reconstruction algorithms and is explained in section 5.4. The studies for hit-finding efficiency and signal-to-noise ratio begin in section 5.2.

The information given by the telescope enables the determination of the location of the hits in the detector. Therefore it is practical to define a cluster of charge around the hit position. The signal-to-noise cut-off value of the cluster is adjusted to optimize hit-finding efficiency, noise suppression and spatial resolution. This is the standard algorithm used for silicon detectors (algorithm A of section 5.4).

A signal-to-noise cut-off value discards the information about the charge collected in some of the strips (those below the threshold), thus limiting the knowledge on the intrinsic resolution of the detector. To understand that, the information from the telescope is used to study how the spatial resolution depends on the charge sharing. The influence of the coupling of a readout strip to its neighboring strips and to the backplane is investigated with algorithms $\mathrm{B}$ and $\mathrm{C}$ of section 5.4.

\footnotetext{
${ }^{10}$ We were running parasitically in the beam line.
} 


\section{$5.1 \quad$ Alignment}

A detailed description of the alignment procedure is found elsewhere [14]. To define a reference system, detectors in planes 1 and 3 are fixed at their nominal positions (see Fig. 7). Detectors 3 and 4 (plane 2) and 7 and 8 (plane 4) are then allowed to rotate.

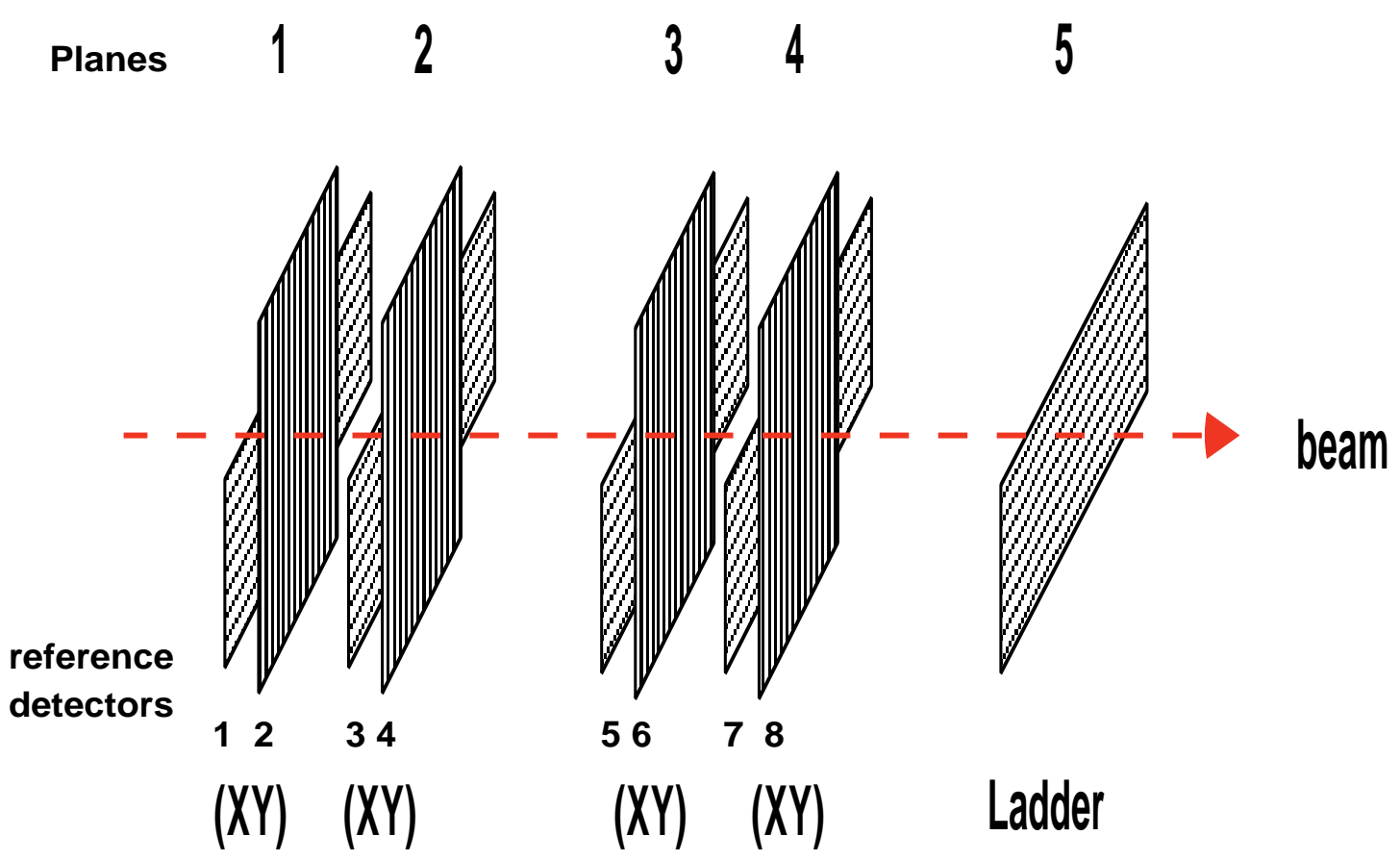

Figure 7: Schematic drawing of the telescope used for the test beam.

The expected hit position is defined as the intersection of the straight line, calculated from hits in the reference planes ( 1 and 3), and the $i^{\text {th }}$ detector. The positions of the detectors which are allowed to rotate, are obtained by performing a minimization of the residuals for each of them. The corrections to the nominal positions are assumed to be infinitesimal.

Residuals in a given detector are obtained using the information from all detectors except that for whose residuals we want to calculate.

The resolution for the $i^{\text {th }}$ detector of the telescope, $\sigma_{i}^{t e l}$, is calculated using the extrapolation errors and the residuals for each detector. The average of all $\sigma_{i}^{\text {tel }}$ gives the resolution of the telescope $\left(\sigma^{\text {avg }}\right)$ which corresponds to $3.0 \mu \mathrm{m}$. The resolution of the telescope is used to calculate the extrapolation error for the plane where the ladder is located, which is equal to $2.8 \mu \mathrm{m}$. 


\subsection{Efficiency and ghosts}

A simple calculation shows that for a readout pitch of $50 \mu \mathrm{m}$, the nominal resolution obtained with a digital readout algorithm is $14.4 \mu \mathrm{m}$. In order to improve on that, we take into account that the charge collected is shared among several strips and define a cluster of charge.

A strip that has the highest signal is taken as a seed (central strip of the cluster), provided its signal is greater than a given number of times its noise. Adjacent strips are added to the cluster if their signals are beyond another threshold value. Both threshold values need to be determined experimentally. The choice of the signal-to-noise threshold for the seed affects the efficiency for detecting a hit. If the threshold value is too low, the hits from noisy channels may be mistaken as real hits. Ghost hits are defined as all spurious hits (noisy channels are included) that appear in a defined region outside that used for the calculation of the efficient hits.

The region to calculate the efficient hits is chosen as $\pm 150 \mu \mathrm{m}$ around the hit position expected from the telescope. This takes into account the angular distribution of tracks caused by multiple scattering and the resolution determined by the distance between the strips. The efficiency for detecting a hit versus the signal-to-noise cut-off value for the central strip is shown in Fig. 8a. Noisy channels are not included in the calculations. The efficiency decreases as the signal-to-noise cut-off value increases.

The percentage of ghost hits versus the signal-to-noise cut-off value for the central strip is shown in Fig. 8b. A given event may have more than one ghost hit. The numbers used to obtain the curve in Fig. 8 are the total number of ghost hits and not the number of events with ghost hits. For a signal-to-noise cutoff value greater than 4 , the number of ghost hits does not change significantly. With a signal-to-noise cut-off value of $4.5 \sigma$ for the central strip in the cluster, an efficiency of $99.8 \%$ is obtained and the number of ghost hits is reduced to about $5 \%$.

\subsection{Charge collection}

Figure 9 displays the total charge collected from eight strips in which hits have been efficiently identified.

A Landau distribution convolved with a Gaussian is used to fit the cluster charge distribution in which the fitted peak position corresponds to the total charge collected. The choice of the algorithm for the clusterization of charge must at least optimize the spatial resolution. Studies of charge collection from the strips were also done with different clusterization schemes (algorithms B and $\mathrm{C}$ in section 5.4). 

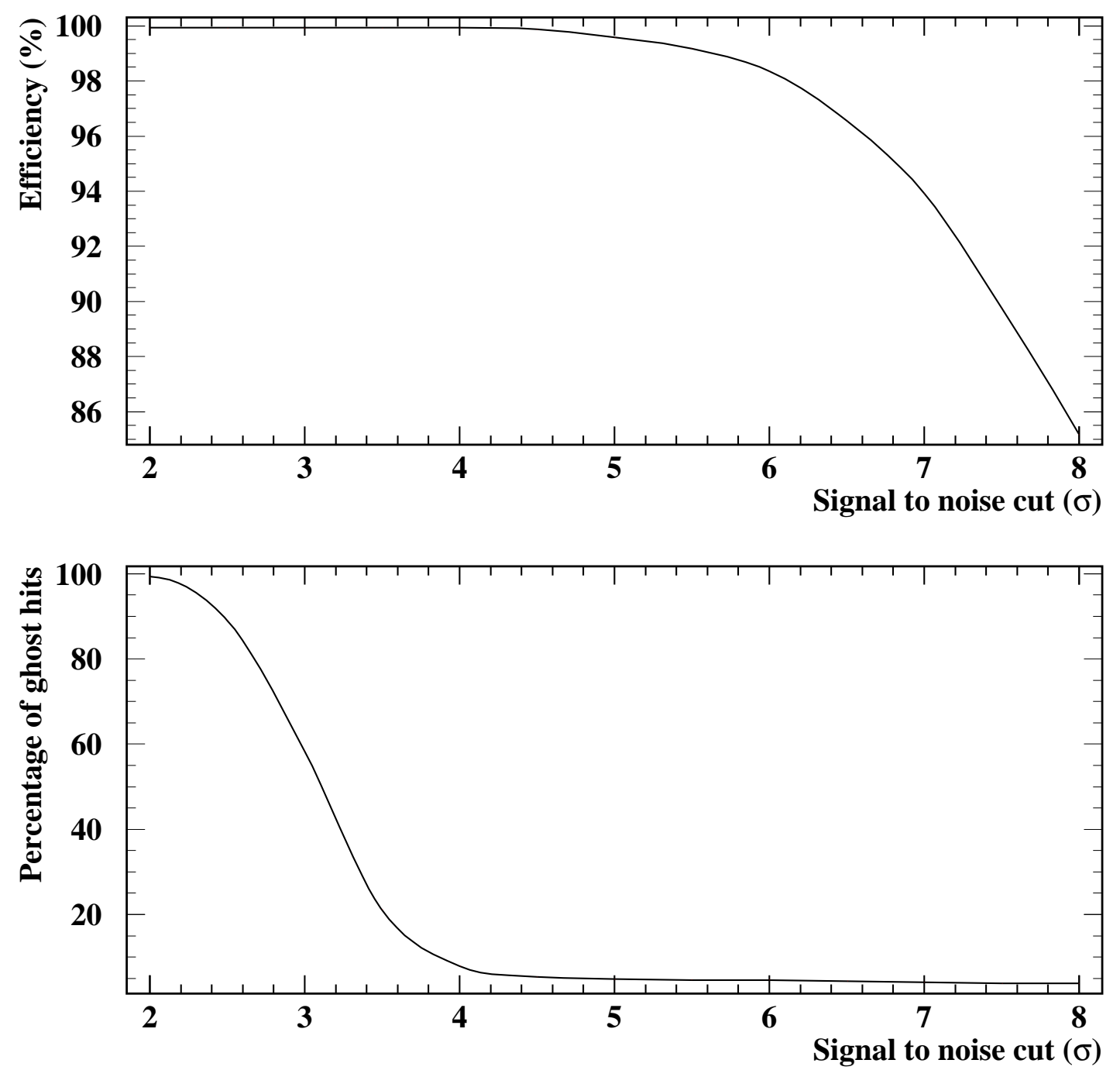

Figure 8: a) Efficiency versus the signal-to-noise cut-off value for the central strip in the cluster, b) percentage of ghost hits versus the signal-to-noise cut-off value for the central strip in the cluster. 


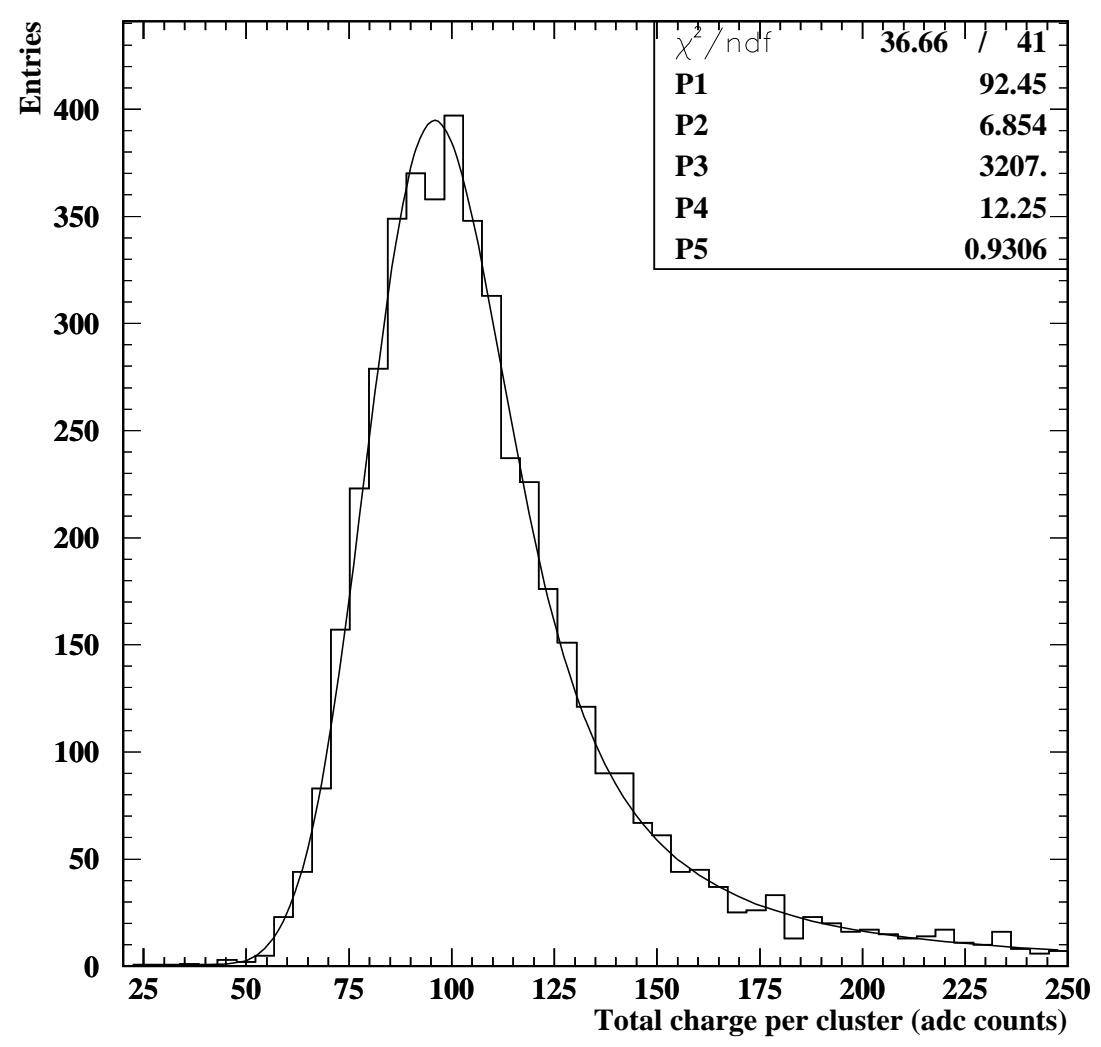

Figure 9: Charge collected from eight strips for efficient hits.

\subsection{Reconstruction algorithms}

We studied three different clusterization schemes

- algorithm A: As discussed in sections 5.2 and 5.3 this is the only practical algorithm for a silicon detector; a signal-to-noise cut-off value for the strips is used to establish a threshold beyond which the charge is recorded.

- algorithm B: A model is used to simulate charge sharing among strips. Assuming this model, the observed pulse heights in eight readout strips are used to calculate the hit positions.

- algorithm C: The previous algorithm can be improved: eight readout strips are used to form a cluster, the total charge is shared among these and the floating strips are also allowed to share charge with the backplane.

As discussed in section 5.2 a signal to noise cut-off value of $4.5 \sigma$ for the central strip was chosen for all three algorithms. The hit positions are calculated from the center of gravity of the strips weighted by their charge.

Figure 10 shows a diagram of the capacitive network of two consecutive readout strips. This diagram is used for algorithms B and C.

Algorithm A: 


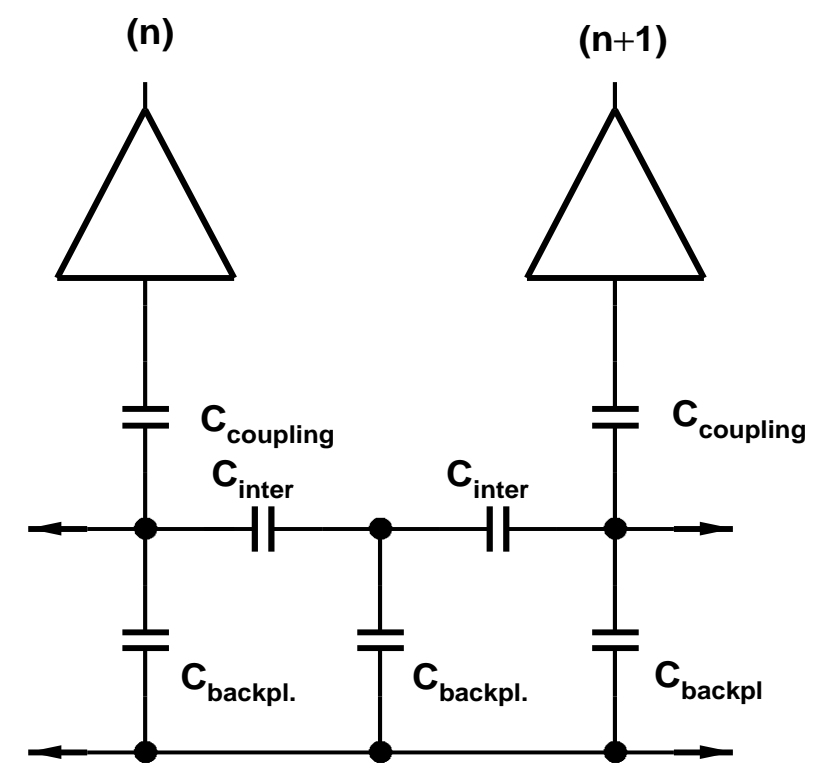

Figure 10: Capacitive network of two consecutive readout strips.

The choice of the threshold for the adjacent strips was given by the value that minimized the residuals. This is shown in table 3 .

\begin{tabular}{|c|c|}
\hline cut-off value for the signal-to-noise ratio $(\sigma)$ & Resolution $(\mu \mathrm{m})$ \\
\hline 1.5 & 7.5 \\
\hline 2.0 & 6.9 \\
\hline 2.5 & 6.6 \\
\hline 3.0 & 6.7 \\
\hline 4.0 & 6.9 \\
\hline
\end{tabular}

Table 3: Cut-off value for the signal-to-noise ratio for the adjacent strips.

The distribution for residuals is displayed in Fig. 11 in which a $2.5 \sigma$ cut-off value for the signal-to-noise ratio was applied for the adjacent strips.

The intrinsic spatial resolution of $6.0 \mu \mathrm{m}$ was obtained by subtracting in quadrature the extrapolation errors $(2.8 \mu \mathrm{m})$ from the residuals $(6.6 \mu \mathrm{m})$.

The corresponding charge distribution is shown in Fig. 12a.

The value for the fitted peak position of Fig. 12a is $13 \%$ less than that of Fig. 9. This suggests that some charge is being lost to the remaining strips which was not taken into account by the clusterization scheme. The same effect is seen in Fig. 12b, which shows the charge distribution for different hit positions between two readout strips.

Algorithm B: 


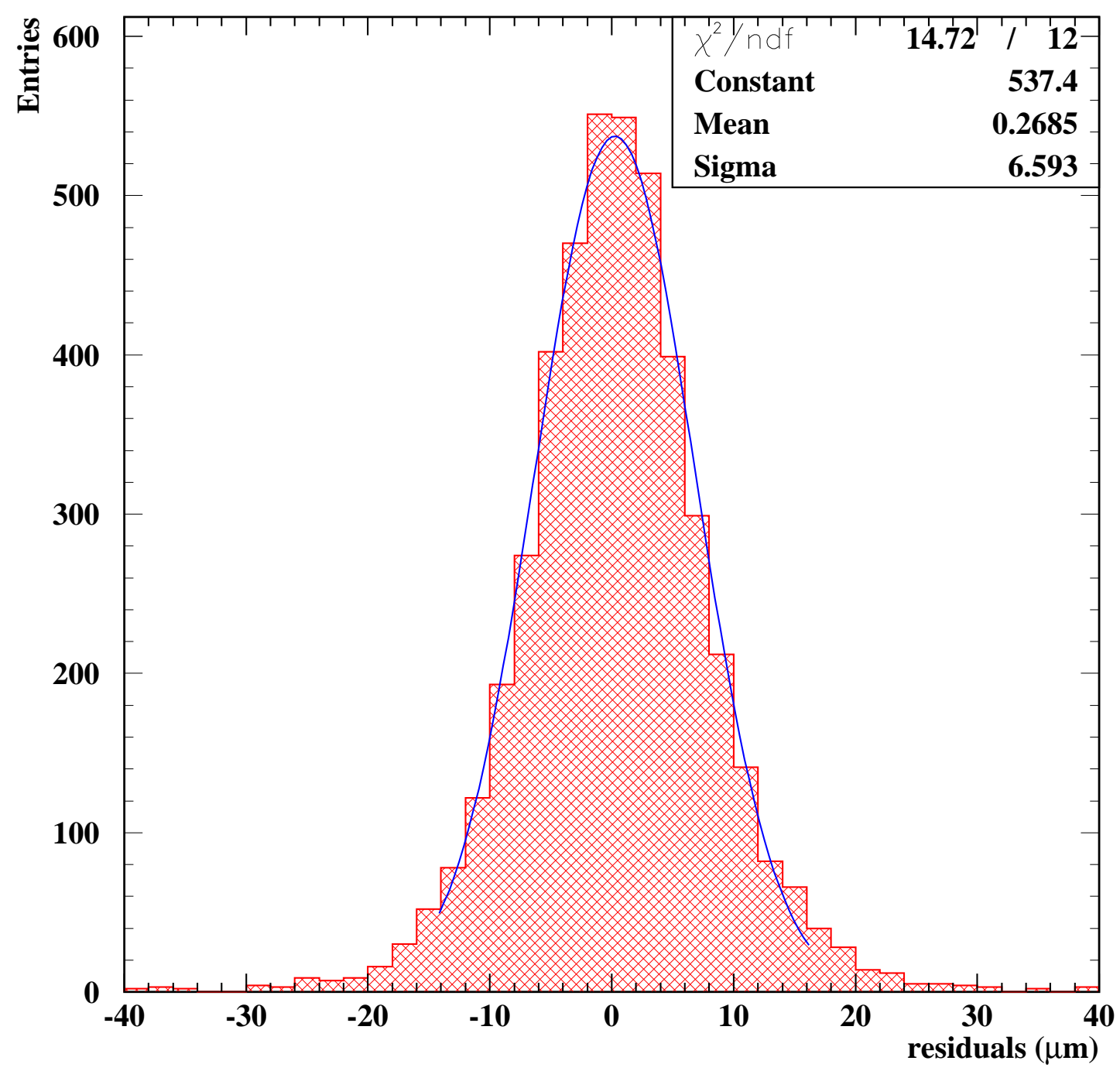

Figure 11: Residuals for algorithm A. 

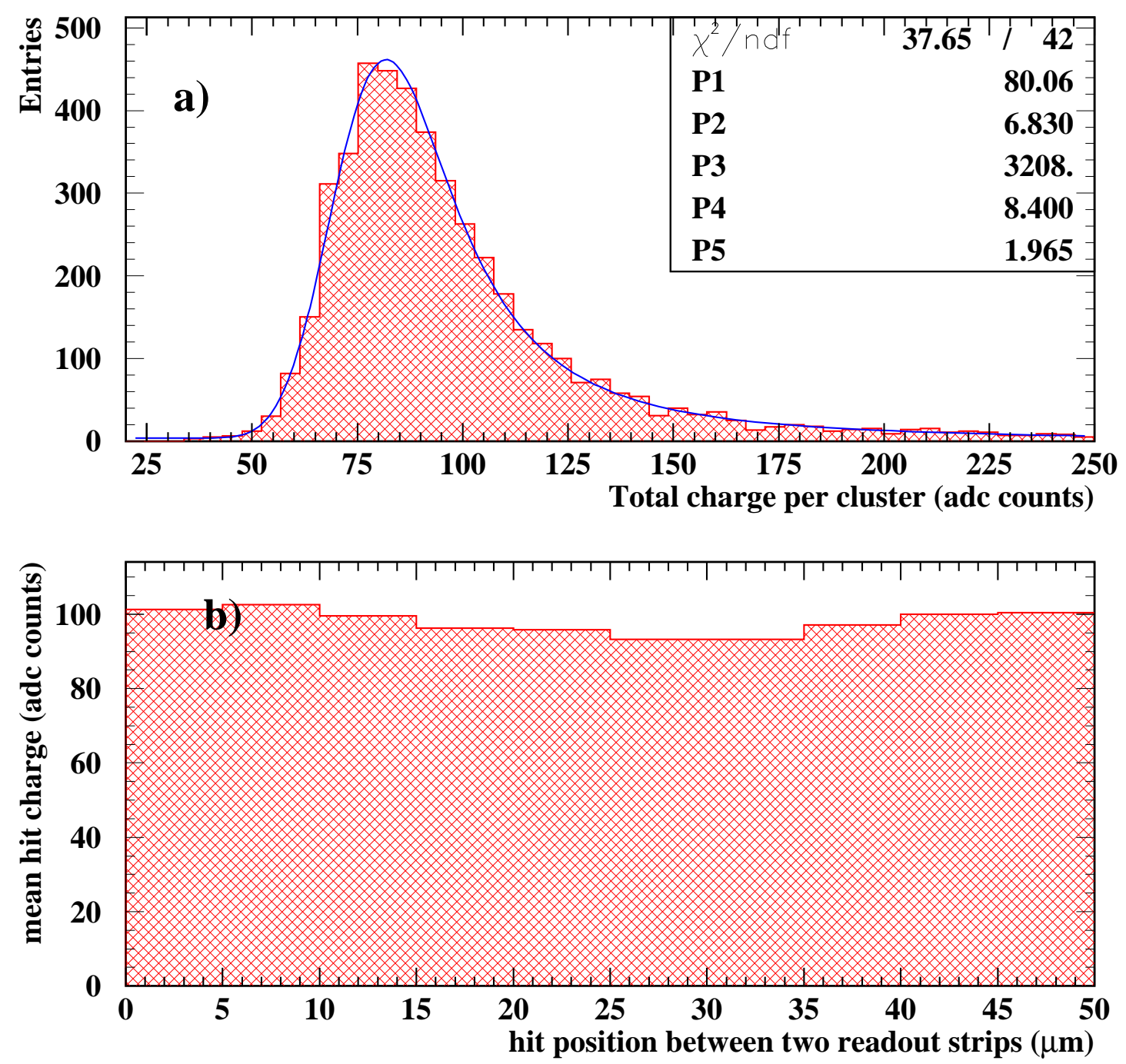

Figure 12: Algorithm A: a) Cluster charge distribution, b) total cluster charge versus hit position between two readout strips. 
In this algorithm we assume that some fraction of the charge is collected via capacitive coupling by eight readout strips. This is represented schematically in Table 4.

\begin{tabular}{|c||c|c|c|c|c|c|c|c|}
\cline { 2 - 10 } \multicolumn{1}{c|}{} & \multicolumn{8}{c|}{ Strip number } \\
\hline steps & 1 & 2 & 3 & 4 & 5 & 6 & 7 & 8 \\
\hline \hline 1 & & & & $Q_{4}$ & $Q_{5}$ & & & \\
\hline 2 & & & $\epsilon Q_{4}$ & $\epsilon Q_{5}$ & $\epsilon Q_{4}$ & $\epsilon Q_{5}$ & & \\
\hline 3 & & $\epsilon^{2} Q_{4}$ & $\epsilon^{2} Q_{5}$ & $2 \epsilon^{2} Q_{4}$ & $2 \epsilon^{2} Q_{5}$ & $\epsilon^{2} Q_{4}$ & $\epsilon^{2} Q_{5}$ & \\
\hline 4 & $\epsilon^{3} Q_{4}$ & $\epsilon^{3} Q_{5}$ & $3 \epsilon^{3} Q_{4}$ & $3 \epsilon^{3} Q_{5}$ & $3 \epsilon^{3} Q_{4}$ & $3 \epsilon^{3} Q_{5}$ & $\epsilon^{3} Q_{4}$ & $\epsilon^{3} Q_{5}$ \\
\hline
\end{tabular}

Table 4: Table for algorithm B.

The hit positions are assumed to be anywhere between the fourth and fifth strip in the cluster. We calculate the total charge in each strip $\left(Q_{4}\right.$ and $\left.Q_{5}\right)$ via a chi-square minimization

$$
\chi^{2}=\sum_{i=1,8}\left(\frac{q_{j}-q_{j}^{\text {meas }}}{\sigma_{j}}\right)^{2},
$$

where the index $j$ corresponds to the readout strips from 1 to $8, q_{j}^{\text {meas }}$ is the measured charge, $q_{j}$ is the expected charge and $\sigma_{j}$ is the noise of the $\mathrm{j}^{\text {th }}$ strip. The charge in each strip is given by

$$
q_{j}=\sum_{k=1,4} \alpha_{k, j}(1-2 \epsilon)
$$

where $\alpha_{k j}$ are the terms obtained from the $k$ rows and $j$ columns of Table 4 .

$$
\alpha_{k j}=\left(\alpha_{k-1, j-1}+\alpha_{k-1, j+1}\right) \epsilon^{k-1},
$$

where $k$ is the number of times the fraction of charge $\epsilon$ is considered (1 to 4 ) and $j$ corresponds to the number of each readout strip (1 to 8 ). The initial conditions $\alpha_{1,4}=Q_{4}, \alpha_{1,5}=Q_{5}$ and $\alpha_{i, k}=0$ for $k \neq 4,5$ imply that the hit position is anywhere between the fourth and fifth strip in the cluster. A minimization procedure gives $\epsilon=0.11$.

The charge distribution is shown in Fig. 13a. As expected, the fitted peak position corresponds to that from Fig. 9. This is because the charge "lost" due to the signal-to-noise cut in algorithm A is now recovered. Figure $13 \mathrm{~b}$ shows the charge distribution for different hit positions between two readout strips.

Clearly, the cluster charge is smaller for hits occurring closer to the floating strip $(25 \mu \mathrm{m})$. This suggests that not all the charge collected by the floating 

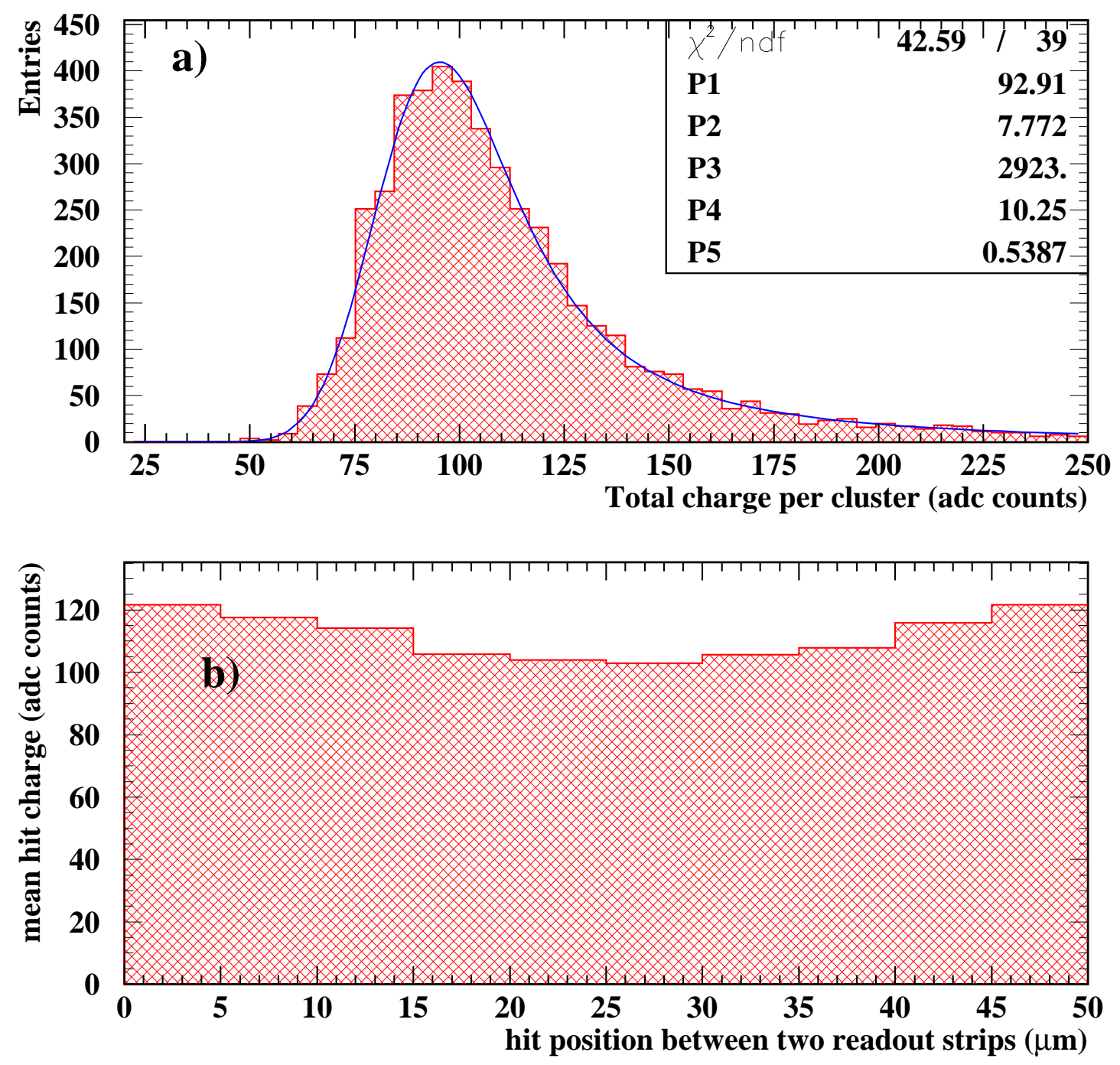

Figure 13: Algorithm B: a) Cluster charge distribution, b) total cluster charge versus hit position between two readout strips. 
strips actually arrive at the readout strips. Probably the charge is being lost to the backplane. Algorithm C investigates this possibility.

\section{Algorithm C}

In this algorithm we assume that some fraction of the charge is collected via capacitive coupling by eight readout strips and the adjacent floating strips. In addition, the floating strips are allowed to share charge with the backplane. This is represented schematically in Table 5 .

\begin{tabular}{|c||c|c|c|c|c|c|c|c|c|c|}
\cline { 2 - 12 } \multicolumn{1}{c|}{} & \multicolumn{9}{c|}{ Strip number } \\
\hline & 1 & $\ldots$ & 5 & 6 & 7 & 8 & 9 & 10 & $\ldots$ & 15 \\
\hline steps & $1_{r}$ & $\ldots$ & $3_{r}$ & $3_{f}$ & $4_{r}$ & $4_{f}$ & $5_{r}$ & $5_{f}$ & $\ldots$ & $8_{r}$ \\
\hline \hline 1 & & & & & $Q_{r}$ & $Q_{f}$ & & & & \\
\hline 2 & & & & $\epsilon Q_{r}$ & $\frac{1}{2} a Q_{f}$ & $\epsilon Q_{r}$ & $\frac{1}{2} a Q_{f}$ & & & \\
\hline 3 & & & $\frac{1}{2} \epsilon a Q_{r}$ & $\frac{1}{2} \epsilon a Q_{f}$ & $\epsilon a Q_{r}$ & $\epsilon a Q_{f}$ & $\frac{1}{2} \epsilon a Q_{r}$ & $\frac{1}{2} \epsilon a Q_{f}$ & & \\
\hline$:$ & & & & & & & & & & \\
$:$ & & & & & & & & & & \\
\hline 8 & $\frac{1}{16} \epsilon^{3} a^{4} Q_{f}$ & $\ldots$ & $\frac{21}{16} \epsilon^{3} a^{4} Q_{j}$ & $\frac{35}{8} \epsilon^{4} a^{3} Q_{r}$ & $\frac{35}{16} \epsilon^{3} a^{4} Q_{f}$ & $\frac{35}{8} \epsilon^{4} a^{3} Q_{r}$ & $\frac{35}{16} \epsilon^{3} a^{4} Q_{f}$ & $\frac{21}{8} \epsilon^{4} a^{3} Q_{r}$ & $\ldots$ & $\frac{1}{16} \epsilon^{3} a^{4} Q_{f}$ \\
\hline
\end{tabular}

Table 5: Table for algorithm $\mathrm{C}$ where $a=\left(1-\epsilon_{b}\right)$.

The hit positions are assumed to be anywhere between a readout strip $r$ and a floating strip $f$. We calculate the total charge in each strip $\left(Q_{r}\right.$ and $\left.Q_{f}\right)$ via a chi-square minimization similar to that in equation( 6$)$. The charge in each strip is given by

$$
\begin{gathered}
q_{n}=\sum_{k=1,8} \alpha_{k, \frac{n+1}{2}}(1-2 \epsilon) \\
\alpha_{k j}=\left(\alpha_{k-1, j-1}+\alpha_{k-1, j+1}\right) \epsilon \quad \text { for } \quad \mathrm{j}=\text { even } \\
\alpha_{k j}=\left(\alpha_{k-1, j-1}+\alpha_{k-1, j+1}\right) \frac{1}{2}\left(1-\epsilon_{b}\right) \quad \text { for } \quad \mathrm{j}=\text { odd }
\end{gathered}
$$

where $k$ is the number of steps ( 1 to 8 ), $n$ corresponds to the number of each readout strip ( 1 to 8 ) and $j$ is the index for each strip (1 to 15$)$. We assume the initial conditions $\alpha_{1,7}=Q_{r}$ (charge in the seed strip), $\alpha_{1,8}=Q_{f}$ (charge in the strip adjacent to the seed strip) and $\alpha_{i, j}=0$ for $j \neq 7,8$. A minimization procedure gives $\epsilon=0.052$ and $\epsilon_{b}=0.172$.

The charge distribution is shown in Fig. 14a. The fitted peak is slightly higher than that of Fig. 9, and this is due to the fact that we account for the backplane charge not observed experimentally. Figure 14b shows the charge distribution for different hit positions between two readout strips. As expected, the distribution is flat. 

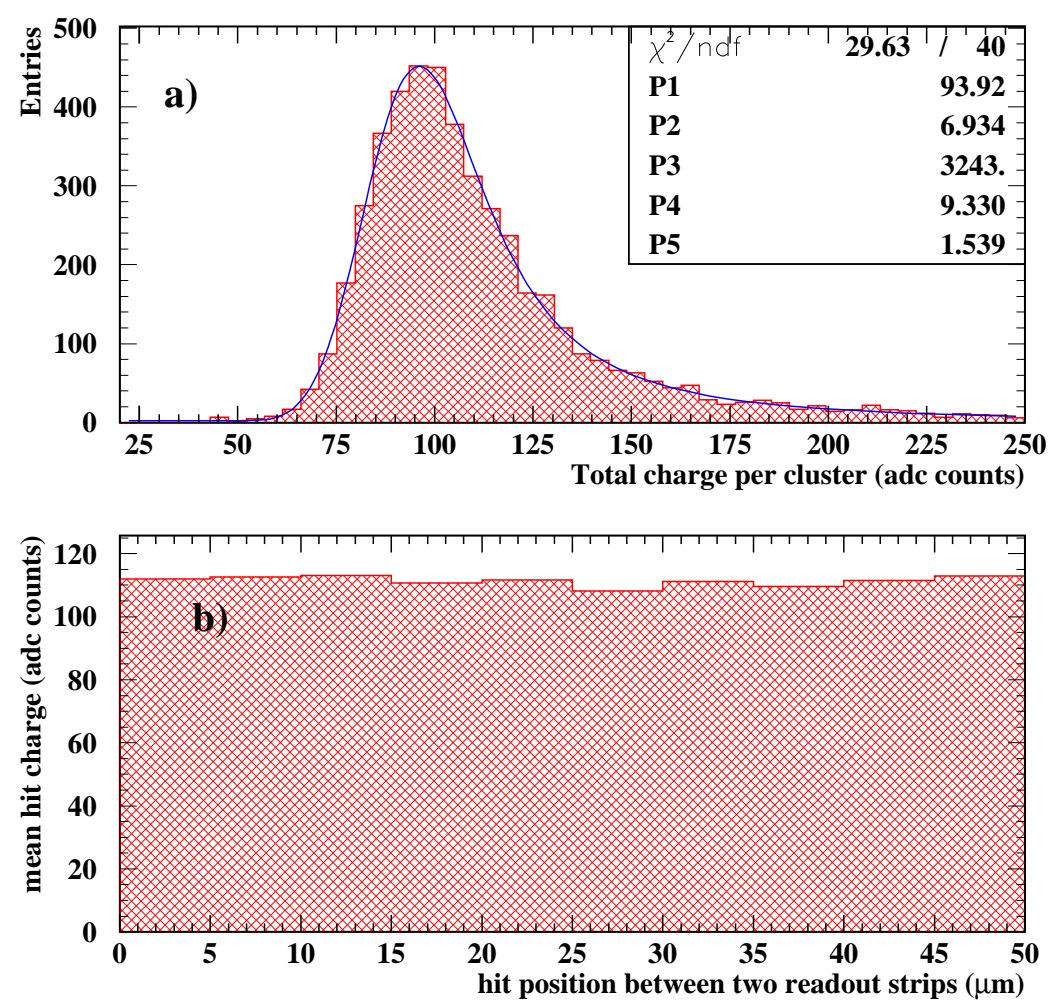

Figure 14: Algorithm C: a) Cluster charge distribution, b) total cluster charge versus hit position between two readout strips.

\section{Conclusions}

Modules of twelve detectors were built for the Silicon Target (STAR) that has been installed in the NOMAD spectrometer.

A detailed comparison between measurements and model predictions for different signal-to-noise ratios has been presented. Laboratory measurements with a radioactive source indicate that ladders of $72 \mathrm{~cm}$ long exhibit a signal-to-noise ratio of the order of 16 .

For long ladders of low leakage current silicon detectors, the dominant contribution to the noise is a function of the resistance and the capacitance of the metal strips. An improvement can be achieved by optimizing the detector design.

For a module with nine detectors we have measured in a test beam a signalto-noise ratio of 19, a hit-finding efficiency of $99.8 \%$ and an intrinsic resolution of $6.0 \mu \mathrm{m}$.

Charge collection varies significantly from the readout to the floating strips.

\section{Acknowledgements}

We would like to thank Tadej Mali for the help with the test beam measure- 
ments. Special thanks to John Conway, Dirk Meyer and Wojciech Dulinski not only for allowing us to use the Strasbourg telescope but also for the support provided. We are indebted to the OPAL and CMS groups for providing an incredible support including the laboratory infrastructure. Thanks to Bernd Schmitt for the help and discussions during detector characterization measurements. Thanks to Kasper "always ready to bond" Mühleman, Rodolfo Gonzalez, Ilia Krassine, José Mulon, Ioannis Papadopoulos and Daniel Voillat. Last but not least thanks to the support of the NOMAD institutions.

\section{References}

[1] CHORUS Collaboration, M. de Jong et al., CERN-PPE/97-33 (1997), A new search for $\nu_{\mu}\left(\nu_{e}\right) \leftrightarrow \nu_{\tau}$ oscillation, CERN-PPE/93-131 (1993).

[2] NOMAD Collaboration, P. Astier et al., CERN-PPE/97-59 (1997), CERNSPSLC/91-21 (1991), CERN SPSLC/9148, SPSLC/P261 Add.1.

[3] J.J. Gomez-Cadenas, J.A. Hernando and A. Bueno, Nucl. Instr. and Meth. A378 (1996) 196.

[4] J.J. Gomez-Cadenas and J.A. Hernando, Nucl. Instr. and Meth. A381 (1996) 223-235.

[5] A. Ereditato, G. Romano and P. Strolin, INFN Napoli Internal Report, 16 February 1996, and A. Ereditato, G. Romano and P. Strolin, CERNPPE/106-96, to be published in Nucl. Instr. and Meth.

[6] A.S. Ayan et al., CERN-SPSC/97-5,SPSC/I213, March , 1997.

[7] J.J. Gomez-Cadenas, Towards a Next Generation $\nu_{\mu}\left(\nu_{e}\right) \leftrightarrow \nu_{\tau}$ Oscillation Search Experiment, Proceedings of the Faro Workshop, Faro, Portugal, September 1996. To be published by World Scientific Eds.

[8] J.J. Gomez-Cadenas, Nucl. Inst. and Meth. A 386 (1997) 66, D. Ferrère, Development of a Silicon Tracker for Neutrino Physics, Proceedings of the $5^{\text {th }}$ International Conference on Advanced Technology and Particle Physics, Como, Italy, October 1996. To be published in Nuclear Physics B.

[9] A. Zalewska, "The Silicon Tracker in the DELPHI Experiment at LEP2", EPS-HEP Conference, Pa 17, Jerusalem, 19-26 August, 1997.

[10] P.P. Allport et al., Nucl. Inst. and Meth. A 310 (1991) 155.

[11] O. Toker et al., Nucl. Instr. and Meth. A 340 (1994) 572.

[12] Orhan Toker, private communication. 
[13] R. Turchetta, Ph.D. Thesis, CERN/HE-91-07, 1019, CNRS, Strasbourg.

[14] A. Cervera-Villanueva et al., Performance of Long Modules of Silicon Microstrip Detectors, NOMAD internal note, MEMO-97-046, 18 November, 1997.

[15] I. Kipnis, Noise analysis due to Strip Resistance in the ATLAS SCT Silicon Strip Module, LBL Internal Report, LBNL-39307, August 1996.

[16] E. Nygard et al., Nucl. Instr. and Meth. A 301 (1991) 506. 


\section{Appendix}

\section{A Calculation of the equivalent noise charge (ENC)}

The steps to calculate the Equivalent Noise Charge are

1. Calculate the output rms noise voltage of a noise source.

2. Translate the output-noise voltage to an equivalent input-noise voltage (using the filter transfer function).

3. Convert the equivalent noise voltage to an equivalent noise charge by using the relation $Q=C_{t} V\left(C_{t}\right.$ is the total input capacitance).

To limit the bandwidth and thus the noise, the signal from the input stage of the amplifier has to be filtered. The VA1 readout chip features a simple CR-RC shaper, whose transfer function is given by

$$
H(s)=\frac{s / \omega_{c}}{\left(1+s / \omega_{c}\right)^{2}},
$$

where $\omega_{c}=1 / T_{p}$ and $T_{p}$ is the peaking time of the shaper.

Multiplying equation (12) by $1 / s$ and taking the inverse Laplace transformation we obtain an equation as a function of time given by

$$
V_{o}(t)=\frac{\omega_{c} t}{\left(e^{\omega_{c} t}\right)} \Delta V_{i}
$$

where $V_{o}$ is the output voltage pulse for a given input voltage step $\Delta V_{i}$ and $e=2.718$. We can calculate the peak voltage $\left(V_{p}\right)$ by requiring $t=T_{p}$,

$$
V_{p}=V_{o}\left(t=T_{p}\right)=\frac{\Delta V_{i}}{e} .
$$

The total mean square noise voltage on the output after filtering is given by

$$
\bar{V}_{o}^{2}=\int S_{n}(\omega)|H(\omega)|^{2} d \omega,
$$

where $S_{n}$ is the noise voltage spectral density at the input. The equivalent noise at the input $V_{i n}$ is amplified by the same factor as the input signal $\Delta V_{i}$. Thus, squaring equation (13) we obtain

$$
\bar{V}_{i n}^{2}=e^{2} \bar{V}_{o}^{2}
$$

Therefore the equivalent noise charge expressed in terms of the number of electrons becomes

$$
\mathbf{E N C}=\frac{C_{t} e}{q}{\sqrt{\bar{V}_{o}}}^{2} \quad r m s e^{-} .
$$




\section{A.1 Series noise}

As an example we consider the noise due to a series resistor at the input of the preamplifier. The voltage spectral density of a resistor is of a Johnson-type

$$
S(\omega)=\frac{2 k T R_{s}}{\pi}
$$

where $R_{s}$ is the equivalent input resistance to the amplifier. The output noise is obtained from equation (15)

$$
\bar{V}_{o}^{2}=\frac{k T R_{s}}{2 T_{p}}
$$

and from equation (18) we obtain

$$
\mathbf{E N C}_{\text {leak }}=\frac{C_{t} e}{q} \sqrt{\frac{k T R_{s}}{2 T_{p}}} \quad r m s e^{-} .
$$

where $C_{t}$ is the equivalent capacitance at the input of the amplifier. To calculate $R_{s}$ and $C_{t}$ for a silicon microstrip detector, we assume each readout channel to be a distributed $\mathrm{RC}$ line as shown in Fig. 15. Each section of this RC line corresponds to an equivalent circuit with a series resistance $R_{l}$ representing the metal strip resistance per unit length and a shunt capacitance $C_{l}$ representing the capacitance (dominated by the interstrip capacitance) per unit length.

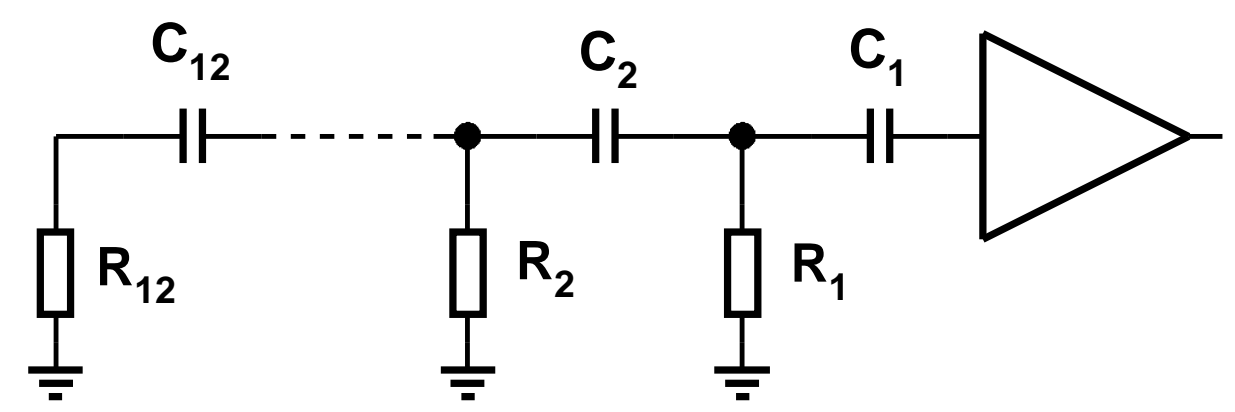

Figure 15: Equivalent circuit used to evaluate the resistance of the metal traces.

For the analysis of the amplifier noise we are interested in evaluating the equivalent impedance at the input of the amplifier, i.e., the input impedance of a distributed RC line when its output is open-circuited. As discussed by Kipnis [15], the equivalent impedance is

$$
Z_{e q}=\frac{1}{\sqrt{s}} \sqrt{\frac{R}{C}} \operatorname{coth}\left(l \sqrt{\frac{R C}{s}}\right),
$$


where $l$ is the total length of the line, $R$ is the resistance per unit length and $C$ is the capacitance per unit length. For $s=j w$, where $j$ is a number in the complex plane, the equivalent series resistance and capacitance can be written as

$$
\begin{aligned}
R_{e q} & =\frac{R_{l}}{x} \cdot \frac{\sinh x-\sin x}{x \cosh x-\cos x}, \\
C_{e q} & =\frac{2 C_{l}}{x} \cdot \frac{\cosh x-\cos x}{x \sinh x+\sin x},
\end{aligned}
$$

where $x=l \sqrt{2 \omega R C}$.

Performing a Taylor series expansion of equations (22) and (23) in the range $0.1<x<1$ one obtains

$$
\begin{aligned}
& R_{e q}=\frac{R_{l}}{3} \text { for } 0.1<x<1 \\
& C_{e q}=\frac{C_{l}}{x} \text { for } 0.1<x<1 .
\end{aligned}
$$

The analytical results from equations (24) indicate a decrease in the total equivalent resistance. These results have also been checked with SPICE simulations using the simple RC model depicted in Fig. 15.

Therefore, one can write equation (20) as

$$
\mathbf{E N C}_{\text {leak }}=\frac{C_{t} e}{q} \sqrt{\frac{k T R_{m s}}{6 T_{p}}} \quad r m s e^{-},
$$

where $R_{m s}$ is the resistance of the metal strip and $C_{t}$ is the total capacitance of the detector, dominated by the interstrip capacitance.

\section{A.2 Parallel noise}

It has been shown that there are two sources of parallel noise: the leakage current and the biasing resistors. These contributions are expressed in terms of the spectral density as,

$$
S_{\text {leak }}(\omega)=\frac{K I_{\text {leak }}}{\pi C_{t}^{2} \omega^{2}}
$$

and

$$
S_{\text {res }}(\omega)=\frac{2 k T}{\pi C_{t}^{2} \omega^{2} R_{p}},
$$

where $I_{\text {leak }}$ is the detector leakage current and $R_{p}$ is the parallel combination of $R_{b}$ (the detector bias resistor) and $R_{f}$ (the feedback resistor across the amplifier). Using the same procedure as discussed in section A.1, we obtain the noise contribution from the leakage current

$$
\mathbf{E N C}_{l e a k}=\frac{e}{q} \sqrt{\frac{q I_{l e a k} T_{p}}{4}}
$$


and from the polarization resistors

$$
\mathbf{E N C}_{r e s}=\frac{e}{q} \sqrt{\frac{k T T_{p}}{2 R_{p}}} .
$$

\section{A.3 Preamplifier noise}

A similar procedure can be used to compute the noise due to the preamplifier and the flicker noise. For completeness we quote the values obtained by Nygard [16] for the flicker noise

$$
\mathbf{E N C}_{1 / f}=\frac{C_{t} e}{q} \sqrt{\frac{F_{k}}{2 W L_{e f f}}},
$$

the transistor channel noise

$$
\mathrm{ENC}_{c h}=\frac{C_{t} e}{q} \sqrt{\frac{k T}{3 g_{m} T_{p}}},
$$

and the transistor bulk-resistance noise

$$
\mathbf{E N C}_{b u l k}=\frac{C_{t} e}{q} \sqrt{\frac{R_{b u l k} \eta^{2} k T}{2 T_{p}}}
$$

where,

- $e=2.718$,

- $q$ is the electron charge in Coulombs,

- $I_{\text {leak }}$ is the leakage current per strip in nanoamperes,

- $T_{p}$ is the peaking time of the shaper in $\mu \mathrm{s}$,

- $k$ is the Boltzmann constant,

- $T$ is the temperature in Kelvin,

- $R_{p}$ is the total parallel resistance at the input of the amplifier,

- $C_{t}$ is the total capacitance at the input of the amplifier,

- $R_{m s}$ is the total resistance from the metal strip,

- $F_{k}$ is a process-dependent constant (typically very small, of the order of $\left.10^{-22}[16]\right)$, 
- $W$ is the transistor width,

- $L_{e f f}$ is the effective transistor length,

- $g_{m}$ is the transconductance,

- $R_{\text {bulk }}$ is the bulk resistance,

- $\eta$ is the ratio between the bulk-to-channel and gate-to-channel transconductances (typically of the order of 0.1 [16].).

Due to the small values of $F_{k}$ and $\eta$, the contributions from flicker and bulk resistance noise can be ignored. Note that the dominant noise source is the channel noise, which is proportional to the total capacitance at the input of the preamplifier and decreases with $1 / \sqrt{g_{m} T_{p}}$. Thus, for our application, since the very long ladder implies a large capacitance, one needs a preamplifier whose input transistor has a large transconductance, and at the same time one needs to shape for a long time. Due to the low value of $\eta$ the bulk resistance term is negligible in the VA1 chip. Instead of using equations (31), (32) and (33) to estimate the noise performance of the VA1 chip, we used the fitted curve obtained from the measured values displayed in Fig. $2 \mathrm{~b}$ of section 3.3. 